\title{
Universal Fluctuations of the S\&100 Stock Index Returns
}

\author{
Rui Gonçalves* and Alberto Pinto ${ }^{\dagger}$ \\ ${ }^{*}$ Faculdade de Engenharia do Porto, R. Dr. Roberto Frias 4200-465, Portugal \\ email: rjasg@fe.up.pt \\ ${ }^{\dagger}$ Dep.to de Matemática da Universidade do Minho, Campus de Gualtar, Braga, Portugal \\ email:aapinto1@gmail.com
}

\begin{abstract}
We analyze the constituents stocks of the well known Standard \& Poor's 100 index (S\&P100) that are traded in the NYSE and NASDAQ markets. We observe the data collapse of the histogram of the S\&P100 index fluctuations to the universal non-parametric Bramwell-Holdsworth-Pinton (BHP) distribution. Since the BHP probability density function appears in several other dissimilar phenomena, our result reveals an universal feature of the stock exchange markets.
\end{abstract}

Keywords: Econophysics, Dynamical systems, Universality, Finance, Econometrics, Stock Exchange Markets

PACS: 89.65.Gh; 05.45.-a;05.40.-a

\section{INTRODUCTION}

The first paper relating statistical laws in physics and in Social Sciences was written by the italian physicist Ettore Majorana [17]. In the paper, Majorana states that the formal analogy between the statistical laws of physics and the ones of social sciences credited the opinion that human facts also undergo a rigid determinism. It is well known that the fluctuations in a global measure of systems with a many body system are often assumed to be of a Gaussian form about the mean value [7]. This assumption is generally true if the system in question can be divided into statistically independent microscopic or mesoscopic elements [14], via the central limit theorem. However, in systems with many interacting constituents there is no reason to expect the central limit theorem to apply. Bak, Tang and Wiesenfeld [4] proposed the hypothesis that under very general conditions non-equilibrium systems consisting of many interacting constituents may exhibit universal behavior occurring naturally.

Our object of analysis is the famous $S \& P 100$ stock index. From a statistical physics point of view, one can think that the stock prices and indexes form a non-equilibrium system with interacting constituents (see [13], [16] and [21]). We discover the data collapse of the histogram of the normalized re-scaled S\&P 100 index to the universal non-parametric Bramwell-Holdsworth-Pinton (BHP) [1] distribution.

\section{THE UNIVERSAL NON-PARAMETRIC BHP PDF}

The universal nonparametric BHP pdf was discovered by Bramwell, Holdsworth and Pinton [1]. The universal nonparametric BHP pdf is the pdf of the fluctuations of the total magnetization, in the strong coupling (low temperature) regime for a two-dimensional spin model $(2 \mathrm{dXY})$, using the spin wave approximation. The magnetization distribution, that they found, is named, after them, the Bramwell-Holdsworth-Pinton (BHP) distribution. The BHP probability density function ( $p d f$ ) is given by

$$
\begin{aligned}
& p(\mu)=\int_{-\infty}^{\infty} \frac{d x}{2 \pi} \sqrt{\frac{1}{2 N^{2}} \sum_{k=1}^{N-1} \frac{1}{\lambda_{k}^{2}}} e^{i x \mu \sqrt{\frac{1}{2 N^{2}} \sum_{k=1}^{N-1} \frac{1}{\lambda_{k}^{2}}}} \\
& . e^{-\sum_{k=1}^{N-1}\left[\frac{i x}{2 N} \frac{1}{\lambda_{k}}-\frac{i}{2} \arctan \left(\frac{x}{N \lambda_{k}}\right)\right]} \cdot e^{-\sum_{k=1}^{N-1}\left[\frac{1}{4} \ln \left(1+\frac{x^{2}}{N^{2} \lambda_{k}^{2}}\right)\right]}
\end{aligned}
$$

where the $\left\{\lambda_{k}\right\}_{k=1}^{L}$ are the eigenvalues, as determined in [3], of the adjacency matrix. It follows, from the formula of the BHP pdf, that the asymptotic values for large deviations, below and above the mean, are exponential and double

CP1168, Vol. 2, Numerical Analysis and Applied Mathematics, International Conference 2009

edited by T. E. Simos, G. Psihoyios, and Ch. Tsitouras

(1) 2009 American Institute of Physics 978-0-7354-0708-4/09/\$25.00 
exponential, respectively (in this article, we use the approximation of the BHP pdf obtained by taking $L=10$ and $N=L^{2}$ in equation (1)). As we can see, the BHP distribution does not have any parameter (except the mean that is normalize to 0 and the standard deviation that is normalized to 1) and it is universal, in the sense that appears in several physical phenomena. For instance, the universal nonparametric BHP distribution is a good model to explain the fluctuations of order parameters in theoretical examples such as, models of self-organized criticality, equilibrium critical behavior, percolation phenomena (see [1]), the Sneppen model (see [1] and [5]), and auto-ignition fire models (see [22]). The universal nonparametric BHP distribution is, also, an explanatory model for fluctuations of several phenomenon such as width power in steady state systems (see Bramwell et al. [1]), rivers height and flow (see Bramwell et al. [2], Gonçalves et al. [12] and Dahlstedt and Jensen [6]), Plasma density and electrostatic turbulent fluxes measured at the scrape-off layer of the Alcator C-Mod tokamak (see Milligen [19]), Wolf's sunspot numbers (see Gonçalves, Pinto and Stollenwerk [10]) and the Standard \& Poor's S\&P100 re-scaled stock index and the re-scaled daily returns of its constituent stocks (see Gonçalves and Pinto[9]).

\section{THE DATA COLLAPSE OF THE S\&P100 INDEX RE-SCALED DAILY RETURNS}

The S\&P 100 index is a subset of the S\&P 500 and it is comprised of 100 leading U.S. stocks with exchange-listed options. The constituents of the S\&P 100 represent about 57\% of the market capitalization of the S\&P 500. The stocks in the S\&P 100 are generally among the largest companies in the S\&P 500. In [9], it is discovered the data collapse of the fluctuations of the daily returns of stock prices in the S\&P100 index to the BHP pdf that we pass to describe. We will focus our analysis on the relative first difference of a re-scaling of the index. The time series $S \& P(t)$ to investigate in our analysis is the $S \& P 100$ index from January of 1987 to September of 2008. Following [9], let the re-scaled time series $S \& P_{r}(t)$ of of the $S \& P 100$ index be $S \& P_{r}(t)=(S \& P(t))^{4 / 9}$. Let $S \& P_{\mu}$ be the sample mean and $S \& P_{\sigma}$ be the sample standard deviation of the re-scaled $S \& P 100$ index $S \& P_{r}(t)$. In [9] is defined the normalized re-scaled $S \& P 100$ index by

$$
S \& P_{n r}(t)=\frac{S \& P_{r}(t)-S \& P_{\mu}}{S \& P_{\sigma}} .
$$

In Figure 1, we show the histogram of the normalized re-scaled $S \& P 100$ index $S \& P_{n r}(t)$ with the BHP pdf on top.

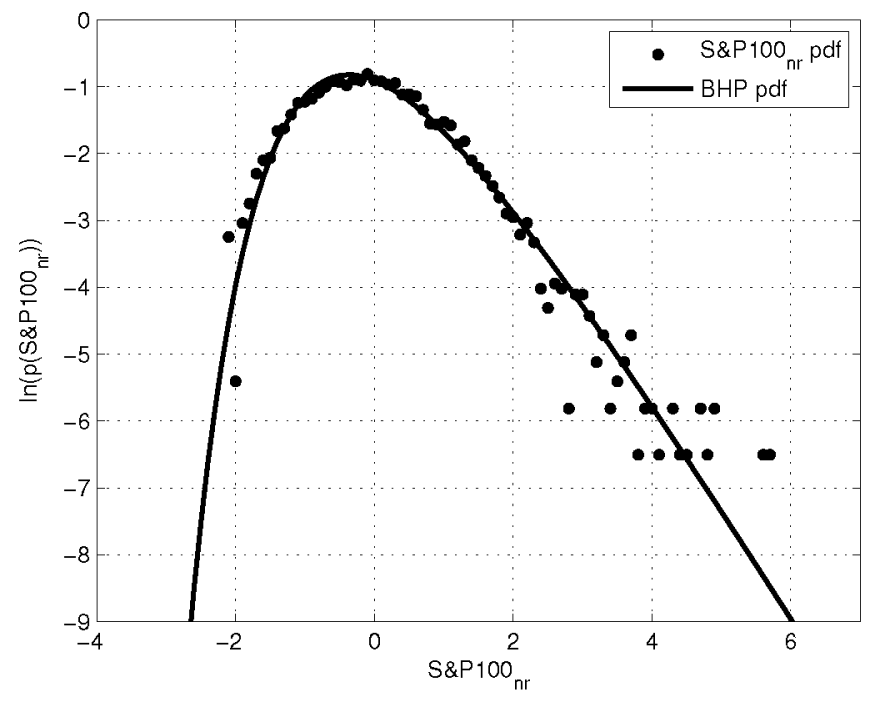

FIGURE 1. Histogram, in the semi-log scale, of the normalized re-scaled $S \& P 100$ index $S \& P_{n r}(t)$ with the BHP pdf on top.

The Kolmogorov-Smirnov (KS) test is a well know non-parametric test of equality of one-dimensional probability distributions used to compare a sample with a reference probability distribution. The Kolmogorov Smirnov statistic quantifies a distance between the empirical cumulative distribution function (cdf) of the sample and the cumulative distribution function of the reference distribution. The null distribution of this statistic is calculated under the null hypothesis that the sample is drawn from the reference distribution. The observed KS statistic for the normalized 
re-scaled $S \& P 100$ index $S \& P_{n r}(t)$ gave a p-value of 0.010441 which is close to the limit value for the conventional criteria for statistical significance $(.001-.05)$.

\section{CONCLUSIONS}

We observe the data collapse of the histogram of the normalized re-scaled S\&P100 index $S \& P_{n r}(t)$ to the universal non-parametric BHP pdf. Since the BHP probability density function appears in several other dissimilar phenomena, our results revealed an universal feature of the stock exchange markets.

\section{ACKNOWLEDGMENTS}

We thank the Programs POCTI and POCI by FCT and Ministério da Ciência, Tecnologia e do Ensino Superior, and Centros de Matemática das Universidades do Minho e do Porto for their financial support.

\section{REFERENCES}

1. S.T. Bramwell, P.C.W. Holdsworth \& J.F. Pinton, Nature, $396552-554$ (1998).

2. S.T. Bramwell, T. Fennell, P.C.W. Holdsworth \& B. Portelli, Europhysics Letters 57310 (2002).

3. S.T. Bramwell, J.Y. Fortin, P.C.W. Holdsworth, S. Peysson, J.F. Pinton, B. Portelli, \& M. Sellitto, Phys. Rev E 63041106 (2001).

4. P. Bak, C. Tang, \& K. Wiesenfeld, Phys. Rev. A 38, 364-374 (1988).

5. K. Dahlstedt \& H. J. Jensen, J. Phys. A: Math. Gen. 34, 11193-11200 (2001).

6. K. Dahlstedt \& H. J. Jensen, Physica A 348 596-610 (2005).

7. B. de Finetti, Theory of Probability, Wiley, 1975.

8. X. Gabaix, G. Parameswaran, V. Plerou \& E. Stanley, Nature 423 267-270 (2003).

9. R. Gonçalves \& A. A. Pinto, arXiv:0810.2508v1 [q-fin.ST] (2008).

10. R. Gonçalves, A. A. Pinto \& N. Stollenwerk, The Astrophysical Journal, 691 (2009), 1583-1586.

11. R. Gonçalves \& A. A. Pinto, Journal of Difference Equations (2009), 1-10. (To be published).

12. R. Gonçalves, H. Ferreira, A. A. Pinto \& N. Stollenwerk, Journal of Difference Equations (2009), 1-8. Special Issue: Dedicated to Saber Elaydi on the Occasion of His 65th Birthday (To be published).

13. P. Gopikrishnan, M. Meyer, L. Amaral \& E. Stanley, The European physical Journal B 3 139-140 (1998).

14. L.D. Landau and E.M. Lifshitz, Statistical Physics, Vol. 1 (Pergamon, 1980).

15. F. Lillo \& R. Mantegna, Intemational Journal of Theoretical and Applied Finance, 3, 405-408 (2000).

16. F. Lillo \& R. Mantegna, Physica A 299 161-167 (2001).

17. E. Majorana, Scientia 36, 58-66 (1942).

18. R. Mantegna \& E. Stanley, Nature 376 46-49 (2001).

19. Milligen, B. Ph. van, Sánchez, R., Carreras, B. A., Lynch, V. E., LaBombard, B., Pedrosa, M. A., Hidalgo, C., Gonçalves, B., Balbín, R. \& W7-AS Team, Physics of Plasmas, 12052507 (2005)

20. V. Pareto, Cours d'Economie Politique, Lausanne and Paris, 1897.

21. V. Plerou, L. Amaral, P. Gopikrishnan, M. Meyer \& E. Stanley Physical Review Letters 837 1471-1474 (1999).

22. P. Sinha-Ray, L. Borda de Água, \& H.J. Jensen, Physica D 157, 186-196 (2001).

23. H. Stanley, V. Plerou \& X. Gabix, Physica A 387 3967-3981 (2008). 